\title{
GARMISCH 2018 - „BACK TO THE ROOTS“
}

\section{Liebe Kolleginnen und Kollegen,}

und wieder neigt sich ein Jahr dem Ende entgegen. In über 70 Kursen waren an die 2000 Teilnehmer zu Weiterbildung und Fortbildung zu betreuen. Die Feedbackbögen zeigten mit einem Punktescore von 9,2 von 10 die hohe Zufriedenheit der Teilnehmer mit der Qualität unserer Ausbildung und sind Ansporn zu weiteren möglichen Verbesserungen.

\section{Neuigkeiten 2017}

Zwei neue Kursformate, Störherd-Kurs und Zungen-Kurs, wurden gut besucht und werden auch in den Folgejahren auf dem Programm stehen.

In diesem Jahr konnten wir die Kollegen Kastner und Ruhnke als weitere Referenten gewinnen. Glücklich über diese Bereicherung wünschen wir beiden Kollegen viel Freude an der weiteren Lehrtätigkeit in unserer Akademie.

\section{Timmendorf 2017 - große Resonanz}

Anfang September trafen sich 150 Kollegen zum Akupunkturkongress am Timmendorfer Strand. Das interessante und vielfältige Programm fand einen regen Zuspruch. Das Thema Niere stellte den roten Faden der Woche dar und die Beiträge zeigten die vielfältigen Interventionsmöglichkeiten, die uns in der Akupunkturarztpraxis zur Verfügung stehen. Auch der kollegiale Austausch kam sowohl in den Workshops als auch beim Gala-Abend nicht zu kurz. Besonders hat es uns - die Organisatoren - gefreut, dass 23 Teilnehmer erstmalig in Timmendorf waren. Dies lässt sich auch auf die Aktion „Ü55 trifft U45“ zurückführen, die erfahrene ältere Kollegen und jüngere Einsteiger in die Akupunktur zusammenbringen soll. An dieser Stelle ganz herzlichen Dank an die „Alten Hasen“, die es hervorragend verstanden haben, die jungen Kollegen zur Teilnahme zu motivieren.

\section{Timmendorf 2018 - Programm zum Jahresende} Für Timmendorf 2018 (09.09.-14.09.2018) haben wir schon viele interessante Referate und Workshops um das Thema „Herz“mit zahlreichen Referenten aus dem In- und Ausland für Sie zusammengestellt. Eine erste Programmvorschau für Sie ist bereits zum Jahresbeginn geplant.

\section{Prüfungsregelung A-Diplom/TCM-Diplom}

Ab 2018 werden die A-Diplom- und TCM-Diplom-Prüfungen im Konsens mit anderen großen Fachgesellschaften aus Qualitätssicherungsgründen wieder mit schriftlicher und praktischer Prüfung durchgeführt. Aufgrund des hohen Aufwandes einer solchen Prüfung erfolgen diese zentral einmal im Jahr in Garmisch. Die B-Diplom-Prüfung ist davon ausgenommen. Diese kann wie bisher im Rahmen von Kurswochenenden abgelegt werden und verlangt die 350 UE und die Vorlage von 10 ausgearbeiteten Behandlungsfällen.

\section{Garmisch 2018}

Nutzen Sie die Gelegenheit, in entspannter Atmosphäre Akupunkturbasics aufzufrischen und/oder sich mit einem Abschluss (A-Diplom/TCM-Diplom) zu qualifizieren. Die Akupunktur erfreut sich ungebremster Beliebtheit bei Patienten und die für 2018 erwartete neue GOÄ (Gebührenordnung für Ärzte) bildet das gesamte Spektrum der Akupunktur und weitererVerfahren der TCM in noch nie dagewesener Weise sehr gut ab.

Die Diplomprüfungen können Sie vom 10.-16. Mai 2018 in Garmisch ablegen. Dazu sollten Sie TCM-Kurse 1-10 absolviert haben. Weitere Informationen können Sie über das Vereinsbüro und unsere Seite (https://www.akupunktur.de/akupunktur/aerzte-zahnaerzte/akupunkturausbildung/tom-diplom.html) erhalten.

Mit guten Wünschen für den Winter, Ihr

\section{B. Ramme}

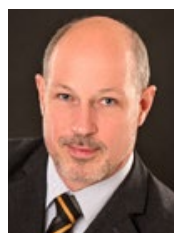

Dr. med. Bernd Ramme

1. Vorsitzender der DAA e.V.,

Osserstraße 40, D-81679 München

Tel. $+4989 / 8145252$

E-Mail des allg. Büros: kontakt@akupunktur.de, Internet:www.akupunktur.de 